\title{
The impact of oral health status on COVID-19 severity, recovery period and C-reactive protein values
}

\author{
Amany Hany Mohamed Kamel, ${ }^{\star 1}$ Ahmed Basuoni, ${ }^{2}$ Zeinab A. Salem ${ }^{3,4}$ and Nermeen AbuBakr ${ }^{1}$
}

\section{Key points}

Oral health status could have a potential impact on the severity of COVID-19.

Poor oral health was correlated to increased values of C-reactive protein.
Delayed recovery period was observed in patients with poor oral health.

\begin{abstract}
Objectives The oral cavity is a potential reservoir for respiratory pathogens which can predispose patients to bacterial super-infection. Several trials have correlated poor oral hygiene with hyper-inflammation. Similarly, COVID-19 severity has been linked to hyper-inflammatory responses. Hence, in this study, we assumed that increased COVID-19 severity may be linked to poor oral health status. This was achieved through assessing oral health status, severity of COVID-19 symptoms, C-reactive protein (CRP) levels and duration of recovery.
\end{abstract}

Methods Cross-sectional study based on a questionnaire; 308 Egyptian patients with confirmed positive polymerase chain reaction (PCR) tests were included in the study after exclusion criteria. The questionnaire was designed with two sections: the first section for oral health evaluation and the second section for COVID-19 severity evaluation. Assessment of the effect of oral health on COVID-19 severity was performed using an oral health score. The effect of oral health on CRP and recovery period were evaluated as secondary endpoints. Data of CRP levels and COVID-19 PCR tests were collected via the questionnaire and confirmed by reviewing medical records.

Results The correlation between oral health and COVID-19 severity showed a significant inverse correlation $(p<0.001$, $r=-0.512$ ). Moreover, the correlation between oral health with recovery period and CRP values also revealed a significant inverse correlation ( $p<0.001,-0.449$ and $p<0.001,-0.190$, respectively), showing that poor oral health was correlated to increased values of CRP and delayed recovery period.

Conclusions Our study provided some evidence that oral health could have a potential impact on the severity of COVID-19. However, the correlation is limited by the study design. A more substantial research project is required to address this relation.

\section{Introduction}

Severe acute respiratory syndrome coronavirus 2 (SARS-CoV-2) is the virus responsible for coronavirus disease 2019 (COVID-19). ${ }^{1}$ The World Health Organisation (WHO) declared a global pandemic on 11 March $2020 .^{2}$ By 17 November 2020, there were over 54 million confirmed cases of COVID-19 with 1,324,249 deaths globally. In Egypt, by this date, there were over 111,009 confirmed cases and 6,465 deaths. ${ }^{3}$

COVID-19 can manifest with a range of symptoms, from mild flu-like symptoms of fever,

'Lecturer, Oral Biology Department, Faculty of Dentistry, Cairo University, Cairo, Egypt; ${ }^{2} \mathrm{MD}$ in Cardiology, Faculty of

Medicine, Cairo University, Cairo, Egypt; ${ }^{3}$ Assistant Professor,

Oral Biology Department, Faculty of Dentistry, Cairo University, Cairo, Egypt. ${ }^{4}$ Assistant Professor, Faculty of Oral and Dental Medicine, Ahram Canadian University, Cairo, Egypt

*Correspondence to: Amany Hany Mohamed Kamel

Email address: amany.hany@dentistry.cu.edu.eg

Refereed Paper

Accepted 14 January 2021

https://doi.org/10.1038/s41415-021-2656-1 dry cough, fatigue, muscle pain and diarrhoea, to more serious presentations characterised by severe pneumonia progressing to adult respiratory distress syndrome (ARDS). Not all affected persons will display symptoms. ${ }^{4}$ The mortality rate of COVID-19 ARDS can approach 40-50\%. ${ }^{5}$ Other cases can be deteriorated to aggressive counteracting of the immune system, known as 'cytokine storm syndrome', in which the levels of released cytokines - tumour necrosis factor (TNF), interleukin-6 (IL-6) and interleukin-1 $\beta$ (IL-1 $\beta$ ) - are injurious to host cells. This may expose patients to an expanded hazard of vascular permeability which can cause damage to many organs, such as the kidneys and heart. ${ }^{5}$ Moreover, vascular complications have also been reported in severe cases. ${ }^{6}$

C-reactive protein (CRP) is a marker of hyper-inflammation. Patients with high levels of CRP have been shown to have a worse prognosis with COVID-19. Recent studies have revealed that the increased CRP levels were likely due to acute inflammatory pathogenesis identified with COVID-19, during which various cytokines were released and their amount was associated with disease severity. ${ }^{7,8}$

Several risk factors for COVID-19 infection have been recognised by the WHO and the Centers for Disease Control and Prevention (CDC), including ageing, diabetes, hypertension, immunodeficiency and cardiovascular diseases. ${ }^{9}$ These comorbidities are associated with increased severity of COVID-19; however, there are various other risk factors that could also be involved in affecting disease outcomes.

Recent studies have demonstrated the association between oral health status and systemic diseases, including systemic infections, cardiovascular disease, pregnancy outcomes and respiratory diseases. ${ }^{10,11}$ Moreover, the impact of good oral care on risk reduction of viral acute respiratory diseases has been reported in numerous studies. ${ }^{12,13}$

The oral cavity is well known as a potential reservoir for respiratory pathogens. It houses more than 700 bacterial species or phylotypes. ${ }^{14}$ Viral respiratory infections predispose patients to bacterial super-infections. It was 
found that severe COVID-19 cases were significantly associated with secondary bacterial infections. ${ }^{15}$ Moreover, several trials have linked COVID-19 severity to high SARSCoV-2 viral load in the nasal and oral cavity. ${ }^{16,17}$

The latest survey conducted by the Egyptian Ministry of Public Health in collaboration with the WHO on the status of oral health among Egyptians noted that $40 \%$ of subjects have encountered dental problems at the time of examination. Visiting behaviours of subjects showed that almost $20 \%$ had not visited a dentist for more than two years, plus another $20 \%$ had never been to a dentist. ${ }^{18}$

The aim of this study was to investigate the potential effect of oral health on COVID-19 illness severity in recovered patients.

\section{Methods}

Sample size

The sample size was 464 recovered COVID19 Egyptian patients. The sample size was calculated from the targeted population (recovered COVID-19 patients) - in Egypt, by the date the study was performed, there were around 20,000. On estimating the sample size, it was 377 , with $95 \%$ confidence level and $5 \%$ confidence interval.

\section{Study design}

The study design was a cross-sectional trial based on a questionnaire survey (Fig. 1). The included patients were recruited from COVID19 dashboard records of the Egyptian Ministry of Health from 1 April 2020 to 1 July 2020. All patients were tested positive for the COVID19 polymerase chain reaction (PCR) test. The questionnaire contained demographic data regarding patient gender, age, weight, height, level of education and general health condition (online Supplementary Material 1). The questionnaire was divided into two sections: the first section for oral health evaluation assessed by a group of dentists (online Supplementary Material 2) and the second section for COVID19 severity assessed by a group of physicians (online Supplementary Material 3). This was done via phone call interviews and/or a link on Google Drive sent to each patient via WhatsApp message. The analysis of the answers to both sections was blinded to the other.

\section{Inclusion criteria}

This included patients aged 19-55 years old, of both genders and with confirmed positive COVID-19 PCR test result.

\section{Fig. 1 A schematic diagram showing the design and methodology of the study}

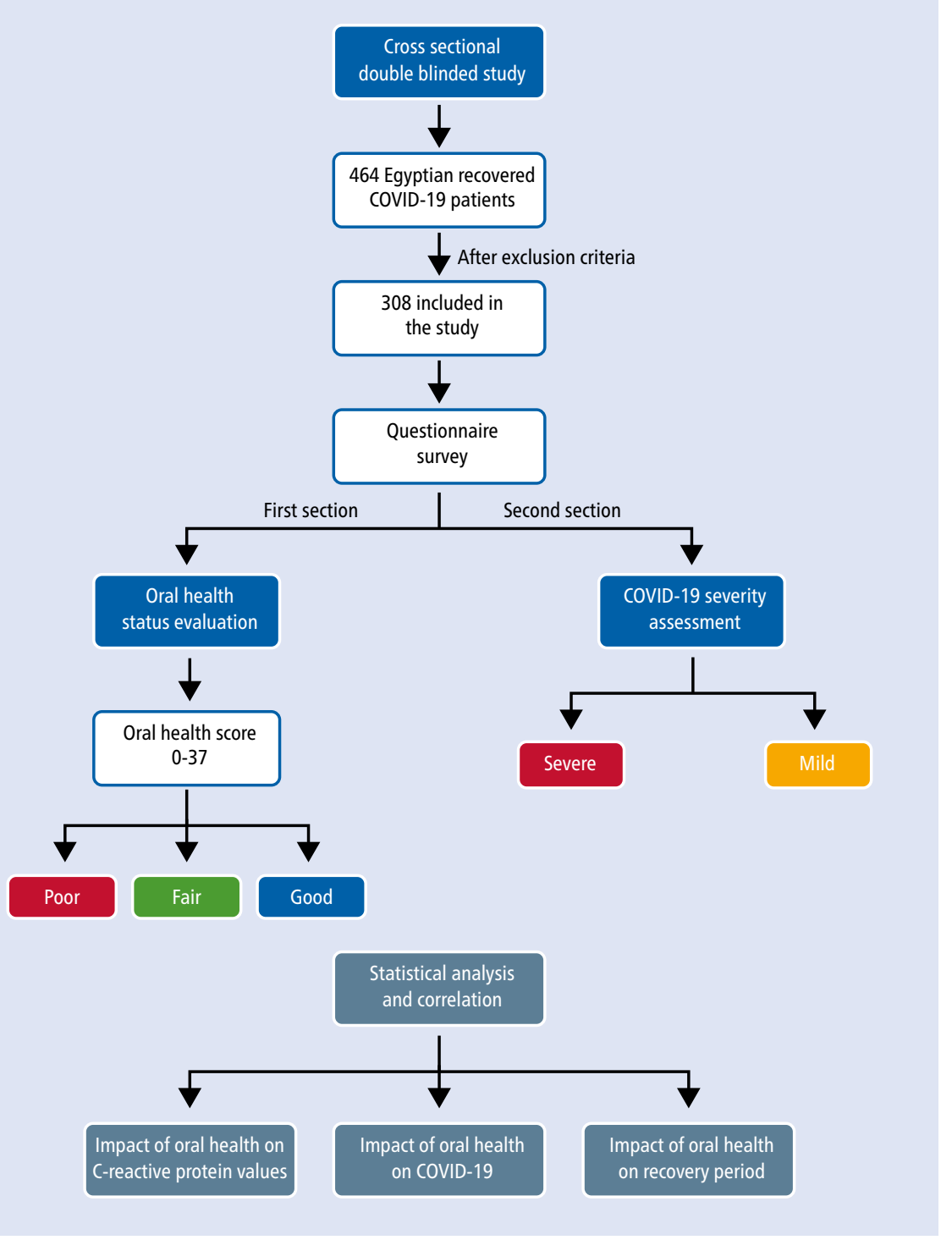

\section{Exclusion criteria}

This included smokers, alcoholics, severe obesity (body mass index $\geq 35$ ), pregnancy and patients with comorbidities (diabetes mellitus, hypertension, cardiovascular diseases, chronic kidney disease, chronic lung diseases and patients who had immunosuppressive conditions or were on immunosuppressive medications). This also included patients who failed to complete the questionnaire or refused to provide consent.

\section{Ethical approval}

This questionnaire and methodology were approved by the Ethics Committee of Faculty of Dentistry, Cairo University, Cairo, Egypt (approval number: 21/6/20). All participants gave their informed consent to the interviewer verbally, using the telephone interview as a format for data collection. In addition, a link to the consent form was sent electronically.

\section{Questionnaire tool}

The questionnaire was reviewed by an expert committee consisting of dental clinicians, physicians and professors at Cairo University. The questionnaire design of the oral health section was guided by a study conducted by Levin et al. (2013) who reported that their questionnaire provided an accurate screening tool for caries risk assessment as reflected by clinical and radiographic examinations, ${ }^{19}$ in addition to Prado et al.'s (2017) questionnaire which was regarded as a reliable and valid tool for evaluating oral health status. ${ }^{20}$ All the questions regarding oral health status in both questionnaires were included in the current questionnaire design and a similar scoring system was utilised. 
Assessment of the effect of oral health on COVID-19 severity as a primary endpoint Oral health interpretation

Interpretation was carried out according to the scores mentioned in online Supplementary Material 2. The questionnaire consisted of 18 questions; each answer denoted a given score of points. The total score ranged from $0-37$ with a higher score denoting good oral health status. The participants were categorised according to their answers into three groups: poor, fair and good oral health, with a threshold of $0-14$, $15-23$ and $24-37$, respectively.

\section{COVID-19 severity interpretation}

Scoring and interpretation were done via the questionnaire. Patients were classified into mild and severe cases. Severe COVID19 illness was characterised by the following criteria: high respiratory rate ( $>30$ breaths per minute); heart rate $>100$ beats/minute; severe dyspnoea or chest pain; oxygen saturation $<93 \%$; and high-grade fever $(>39$ $\left.{ }^{\circ} \mathrm{C}\right)$. Additionally, all hospitalised patients who required oxygen or intensive care unit admission were considered as severe cases. Patients who were not hospitalised due to a shortage of available hospital beds were considered as severe cases if they fulfilled the aforementioned criteria. ${ }^{21,22,23,24}$

\section{Assessment of CRP values}

CRP values for each patient during the first week of illness were obtained retrospectively from laboratory test results supplied to the questionnaire, confirmed by reviewing medical records (online Supplementary Material 3). The inflammatory marker level was then correlated to the COVID-19 severity and oral health status of each patient.

\section{Assessment of recovery period}

Patients were categorised according to their recovery period (complete absence of symptoms) into: fast recovery (two weeks or less); intermediate recovery (four weeks); and delayed recovery (six weeks). Data obtained were then correlated to the COVID-19 severity and oral health status of each participant. This data was obtained from the questionnaire.

\section{Statistical analysis}

Data were coded and entered using the statistical package SPSS version 22 (IBM Corp., Armonk, USA). Categorical variables were summarised as frequency and percentage. Quantitative variables were summarised as

Table 1 Assessment of the effect of oral health of the included participants on COVID-19 severity (primary end point) with a significant difference as $p$ value $<0.001$

\begin{tabular}{|l|l|l|l|l|}
\multirow{2}{*}{ Oral health status } & \multicolumn{3}{c|}{ COVID-19 severity } & \multirow{2}{*}{ P value } \\
\cline { 3 - 4 } & & Severe & Mild & \\
\hline \multirow{2}{*}{ Poor oral health } & N & 52 & 12 & \multirow{2}{*}{$<0.001$} \\
\cline { 2 - 4 } & $\%$ & $65.0 \%$ & $5.3 \%$ & \\
\hline \multirow{2}{*}{ Fair oral health } & N & 20 & 146 & \\
\cline { 2 - 4 } & $\%$ & $25.0 \%$ & $64.0 \%$ & \\
\hline \multirow{2}{*}{ Good oral health } & N & 8 & 70 & \\
\cline { 2 - 4 } & $\%$ & $10.0 \%$ & $30.7 \%$ & \\
\hline & & & & \\
\hline
\end{tabular}

means \pm standard deviation. Comparisons between groups were carried out using the chi-squared test when comparing for the incidence of studied parameters and an unpaired t-test was used when comparing variables between the two groups. Receiver operating characteristic (ROC) curve analysis was done to detect the prediction value of oral health score. Spearman's correlation was used for correlation between studied variables. A p value $<0.05$ was considered significant.

\section{Results}

\section{Survey respondents}

Responses were received from 464 participants, but only 308 respondents were included in the study as 156 respondents were excluded according to exclusion criteria - 4 patients were $<19$ years old, 18 were $>55$ years old, 30 were smokers, 34 were severely obese, 3 were pregnant, 63 had comorbidities and 4 failed to complete the questionnaire or refused to provide consent. See online Supplementary Material 4 for demographic data and online Supplementary Material 5 for descriptive statistics.

Table 2 Correlations between oral health and COVID-19 severity, recovery period and CRP values

\begin{tabular}{|c|c|c|c|c|c|}
\hline \multicolumn{2}{|l|}{ Variable } & Oral health status & COVID-19 severity & Recovery period & CRP \\
\hline \multirow{3}{*}{$\begin{array}{l}\text { Oral health } \\
\text { status }\end{array}$} & $\mathrm{R}$ & 1.000 & $-0.512^{* *}$ & $-0.449 * \star$ & $-0.190^{\star}$ \\
\hline & $P$ value & . & 0.000 & 0.000 & 0.018 \\
\hline & N & 308 & 308 & 308 & 308 \\
\hline \multirow{3}{*}{$\begin{array}{l}\text { COVID-19 } \\
\text { severity }\end{array}$} & $\mathrm{R}$ & $-0.512^{\star *}$ & 1.000 & $-0.575^{\star \star *}$ & $-0.369^{\star *}$ \\
\hline & $P$ value & 0.000 & . & 0.000 & 0.000 \\
\hline & $\mathrm{N}$ & 308 & 308 & 308 & 308 \\
\hline
\end{tabular}

Effect of oral health on COVID-19 severity (primary endpoint)

The incidence of severe COVID-19 illness was significantly observed in participants with poor oral health status $(\mathrm{p}<0.001)$. Participants with good oral health status had a significantly reduced incidence of severe COVID-19 illness ( $\mathrm{p}<0.001, \mathrm{r}=-0.512)$ (Tables 1 and 2; Fig. 2a).

\section{Subgroup analysis of serious COVID-19 cases}

Subgroup analysis of severe COVID-19 cases according to deterioration timing showed $23.75 \%$ and $76.25 \%$ of serious cases deteriorated during the first and second weeks of illness, respectively. The incidence of poor oral health status in those who experienced first-week deterioration (63.1\%) was significantly higher than that of good oral health status $(10.6 \%)(\mathrm{p}<0.001)$. Similarly, the incidence of poor oral health status in those who experienced second-week deterioration (65.6\%) was significantly higher than that of good oral health status $(9.8 \%)(\mathrm{p}<0.001)$ (Table 3). 
Fig. 2 a) A graph showing the impact of oral health status on COVID-19 severity. b) A graph showing the impact of oral health status on the recovery period of COVID-19 patients. c) A graph showing the impact of oral health status on CRP values during the first week of COVID-19 illness. d) ROC curve assessing oral health score as an indicator for COVID-19 severity in the studied population. The area under the curve was found to be 0.828 ( $95 \%$ confidence interval was between 0.745 and 0.911 )

A

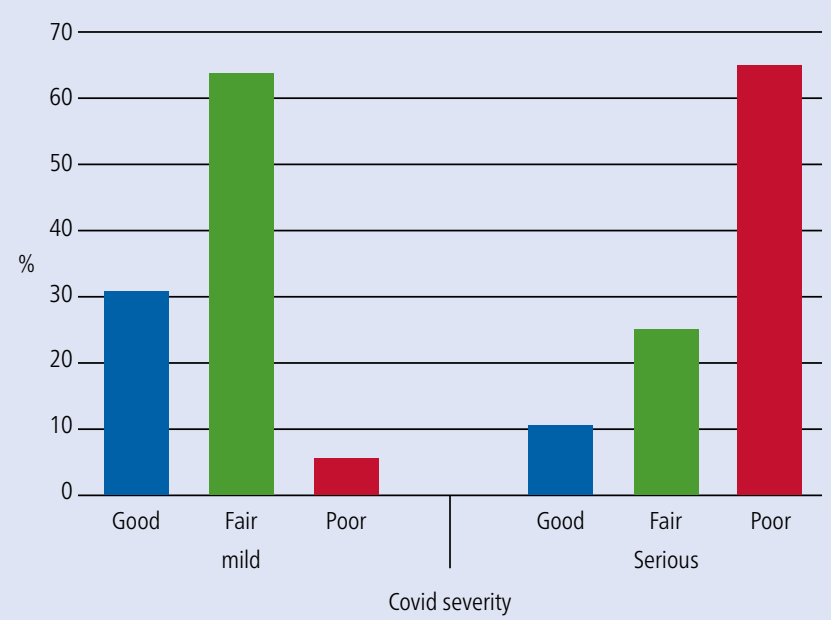

C

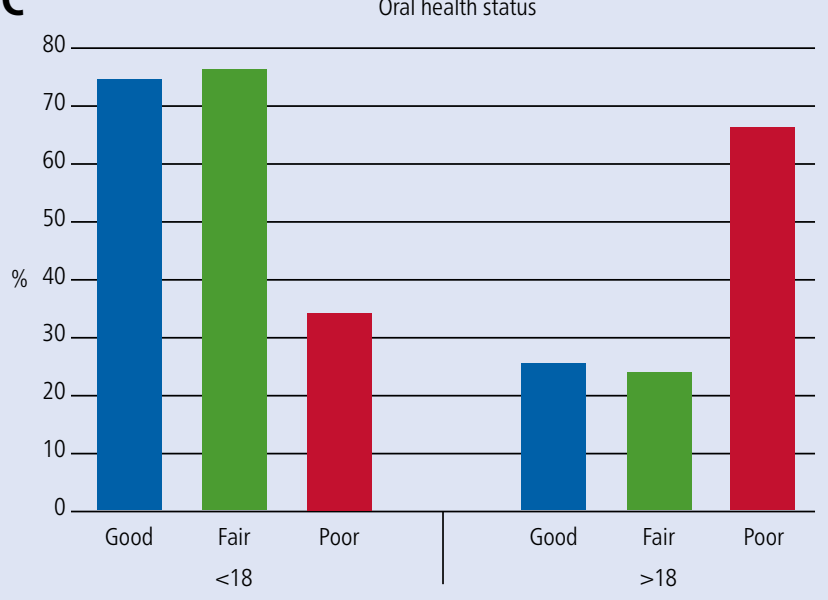

B

Oral health status

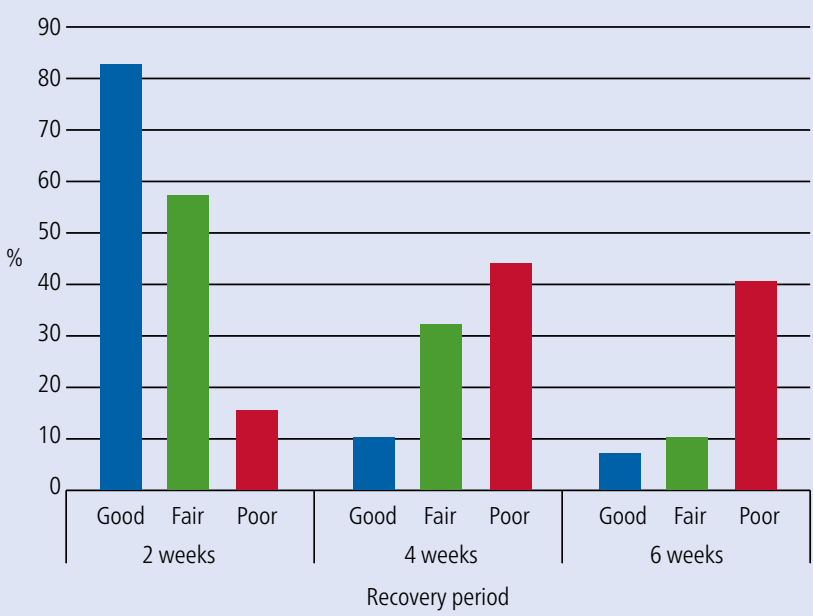

D

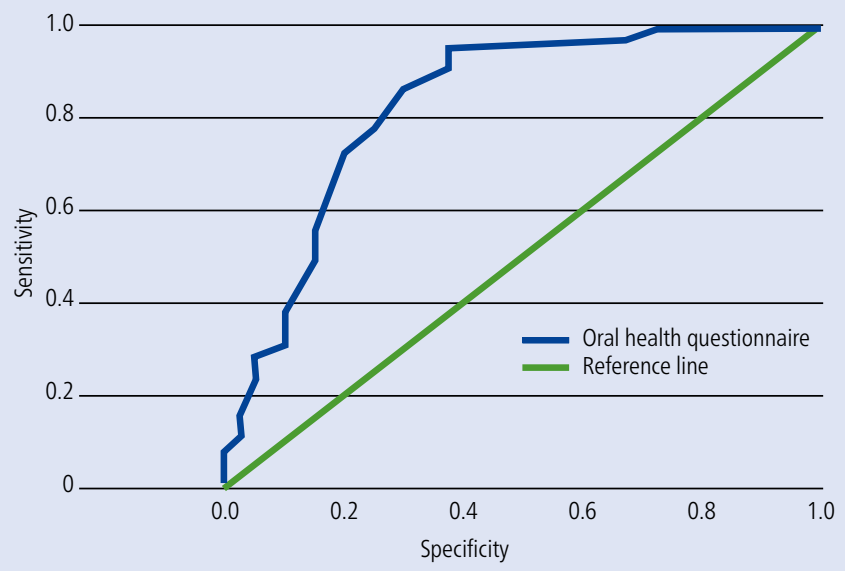

CRP

\section{Secondary endpoints}

Effect of COVID-19 severity on the recovery period

The incidence of the delayed recovery period (six weeks) was significantly higher in severe COVID-19 patients (45.0\%) compared to that in mild cases $(6.1 \%)(\mathrm{p}<0.001)$. Conversely, a fast recovery period (two weeks) was significantly observed in mild COVID-19 cases (70.2\%) compared to that in severe cases $(10 \%)$ $(\mathrm{p}<0.001, \mathrm{r}=-0.575)$ (Tables 2 and 4).

\section{Effect of oral health on the recovery period} The incidence of the delayed recovery period (six weeks) was significantly higher in those with poor oral health status $(40.6 \%)(\mathrm{p}<0.001)$ and a fast recovery period (two weeks) was significantly observed in those with good oral health status $(82.1 \%)(\mathrm{p}<0.001, \mathrm{r}=-0.449)$ (Tables 2 and 4; Fig. 2b).

Effect of COVID-19 severity on CRP values

The incidence of elevated values of CRP ( $>18 \mathrm{mg} / \mathrm{L}$ ) significantly occurred in severe COVID-19 participants $(75.0 \%)(\mathrm{p}<0.001)$ and lower CRP values $(<18 \mathrm{mg} / \mathrm{L})$ were significantly observed in mild COVID-19 participants $(81.6 \%)(\mathrm{p}<0.001, \mathrm{r}=-0.369)$ (Tables 2 and 4).

\section{Effect of oral health on CRP values}

The incidence of elevated values of CRP ( $>18$ $\mathrm{mg} / \mathrm{L}$ ) significantly occurred in those with poor oral health status $(65.6 \%)(\mathrm{p}<0.001)$. Significantly, lower CRP values $(<18 \mathrm{mg} / \mathrm{L})$ were observed in those with good oral health status $(74.4 \%)(\mathrm{p}<0.001, \mathrm{r}=-0.190)$ (Tables 2 and 4; Fig. 2c).

\section{ROC curve analysis}

Oral health score (ROC) curve analysis showed $\mathrm{p}<0.001$; at a cut-off value of 18 for health score with a $71 \%$ sensitivity (true positive cases) and $80 \%$ specificity (true negative cases), positive predictive value (PPV) $77 \%$, negative predictive value (NPV) 75\% (Fig. 2d).

\section{Discussion}

We aimed to investigate the effect of oral health on the severity of COVID-19 illness in recovered patients via a detailed questionnaire, as well as previous access to health data through a nationwide database and blood investigation 
Table 3 Subgroup analysis of COVID-19 severe cases according to deterioration timing with a significant difference as $p$ value $<0.001$

\begin{tabular}{|c|c|c|c|c|c|}
\hline \multirow{2}{*}{\multicolumn{2}{|c|}{ Severe COVID-19 }} & \multicolumn{3}{|c|}{ Oral health status } & \multirow{2}{*}{ P value } \\
\hline & & Poor oral health & Fair oral health & Good oral health & \\
\hline \multirow{2}{*}{$\begin{array}{l}\text { First week } \\
\text { deterioration }\end{array}$} & $\mathrm{N}$ & 12 & 5 & 2 & \multirow{4}{*}{$<0.001$} \\
\hline & $\%$ & $63.1 \%$ & $26.3 \%$ & $10.6 \%$ & \\
\hline \multirow{2}{*}{$\begin{array}{l}\text { Second week } \\
\text { deterioration }\end{array}$} & $\mathrm{N}$ & 40 & 15 & 6 & \\
\hline & $\%$ & $65.6 \%$ & $24.6 \%$ & $9.8 \%$ & \\
\hline
\end{tabular}

results through corresponding participant health records. The analysis of the dental section of the questionnaire was performed by a group of dentists blinded to the results of the COVID-19 severity section (which was analysed by a group of physicians); therefore, proactive efforts to mitigate the risk of bias were made.

Participants with known risk factors and comorbidities that could affect COVID-19 severity other than the investigated parameter (oral health) were excluded. In addition, subjects above 55 years of age were excluded as they are considered to be more prone to experiencing severe COVID-19. ${ }^{25}$

Assessment of the severity of COVID19 patients has been somewhat unclear, but guidelines from different disease centres, such as the CDC, WHO, NHS and National Institute for Health and Care Excellence (NICE), used the same criteria as in this study to classify and assess COVID-19 severity.9, ${ }^{9,226}$ However, all available scores which classified pneumonia or COVID-19 severity were dependent on faceto-face consultations and examination, which were not applicable in the COVID-19 era, and some examination tools have also been prohibited. Some trials showed good results in assessing patients using phone calls, video calls or filling hospital forms. Therefore, using the questionnaire tool to assess COVID-19 severity can be beneficial. ${ }^{27,28,29}$

In the current study, it was observed that the severity of COVID-19 symptoms significantly increased in patients with poor oral health status. Moreover, symptoms of severity significantly decreased in those with good oral health status ( $\mathrm{p}<0.001)$. These findings highlighted the potential impact of oral health status on COVID-19 severity. This is in agreement with several studies that demonstrated the role of oral health in secondary respiratory infections, either bacterial or viral. ${ }^{12,13}$

In patients with poor oral health status, the bacterial count colonising teeth was proven to be raised twofold to tenfold, thus introducing more bacteria into the bloodstream, resulting in bacteraemia. ${ }^{30}$ It was reported that, when a soluble antigen enters the bloodstream, it may interact with a specific circulating antibody and produce an immunocomplex. These macromolecular complexes stimulate various chronic and acute inflammatory reactions. ${ }^{31}$

Moreover, the pro-inflammatory cytokines, such as gamma interferon, interleukins, prostaglandin E2 and TNF, attain increased tissue concentrations in periodontitis. These mediators fight against various microorganisms, but when the immunologic response becomes hyperactive, it can damage various tissues. The periodontium therefore acts as a reservoir of these cytokines, which in turn enter the circulation, induce systemic effects, and affect blood coagulation and platelet function. ${ }^{32}$ In addition, periodontitis as an inflammatory disease may encourage the liver to generate CRP. CRP binds to damaged cells and fixes complement, which in turn enhances phagocytes, mainly neutrophils. ${ }^{33}$

In the same context, it was reported that patients with severe COVID-19 experienced an increased level of CRP compared to mild or moderate cases. In addition, it was shown that the levels of CRP increased tenfold in the patients who died compared to those who recovered. These increased levels of CRP might be linked to the overproduction of inflammatory cytokines which could be aggravated by poor oral health status. ${ }^{34}$

The oral cavity could be a source of viral replication and secondary infections. Teeth are unique in being non-shedding surfaces in the body. This makes them a good environment for the growth and development of dental plaque, especially in those with poor oral hygiene status. A high viral and bacterial load in the oral cavity can worsen various systemic diseases, ${ }^{11}$ revealing the strong link between the body and the oral cavity. Moreover, improper oral hygiene increases the risk of inter-bacterial exchange between mouth and lungs, thus increasing the incidence of respiratory infections and postviral bacterial complications..$^{12,35}$

\begin{tabular}{|c|c|c|c|c|c|c|c|c|c|}
\hline \multirow{2}{*}{\multicolumn{3}{|c|}{ Secondary endpoint }} & \multicolumn{2}{|c|}{ COVID-19 severity } & \multirow{2}{*}{ P value } & \multicolumn{3}{|c|}{ Oral health status } & \multirow{2}{*}{$P$ value } \\
\hline & & & Severe & Mild & & Poor & Fair & Good & \\
\hline \multirow{6}{*}{ Recovery period } & \multirow{2}{*}{ Six weeks } & Count & 36 & 14 & \multirow{6}{*}{$<0.001$} & 26 & 18 & 6 & \multirow{6}{*}{$<0.001$} \\
\hline & & $\%$ & $45.0 \%$ & $6.1 \%$ & & $40.6 \%$ & $10.8 \%$ & $7.7 \%$ & \\
\hline & \multirow{2}{*}{ Four weeks } & Count & 36 & 54 & & 28 & 54 & 8 & \\
\hline & & $\%$ & $45.0 \%$ & $23.7 \%$ & & $43.8 \%$ & $32.5 \%$ & $10.3 \%$ & \\
\hline & \multirow{2}{*}{ Two weeks } & Count & 8 & 160 & & 10 & 94 & 64 & \\
\hline & & $\%$ & $10.0 \%$ & $70.2 \%$ & & $15.6 \%$ & $56.6 \%$ & $82.1 \%$ & \\
\hline \multirow{4}{*}{ CRP } & \multirow{2}{*}{ More than 18} & Count & 60 & 42 & \multirow{4}{*}{$<0.001$} & 42 & 40 & 20 & \multirow{4}{*}{$<0.001$} \\
\hline & & $\%$ & $75.0 \%$ & $18.4 \%$ & & $65.6 \%$ & $24.1 \%$ & $25.6 \%$ & \\
\hline & \multirow{2}{*}{ Less than 18} & Count & 20 & 186 & & 22 & 126 & 58 & \\
\hline & & $\%$ & $25.0 \%$ & $81.6 \%$ & & $34.4 \%$ & $75.9 \%$ & $74.4 \%$ & \\
\hline
\end{tabular}


It was verified that periodontopathic bacteria were present in the metagenome of patients with severe SARS-CoV-2 infection, where elevated concentrations of Fusobacterium, Prevotella and Staphylococcus were detected. ${ }^{36}$ Bacterial superinfections were reported to be high in patients with severe COVID-19 illness, with $>50 \%$ of deaths manifesting bacterial super-infections. ${ }^{37,38}$

There are four possible mechanisms that could explain how oral bacteria could be involved in the pathogenesis of respiratory infections: 1) aspiration of oral pathogens into the lungs; ${ }^{12}$ 2) periodontal disease-associated enzymes could facilitate colonisation and adherence of various respiratory pathogens to the airways; 3) under specific conditions, the dental plaque could harbour colonies of pulmonary pathogens and promote their growth; ${ }^{39}$ and 4 ) in untreated periodontal disease, a large variety of cytokines are continuously released which may alter the respiratory epithelium, making it more prone to get infected with respiratory pathogens. ${ }^{40}$

Several trials have linked COVID-19 severity to viral load. ${ }^{17,18}$ Viral loads are not only high in the nasal cavity and nasopharynx but also in the oropharynx, making the oral cavity a rich source of potentially infectious viruses. Wölfel et al. (2020) reported active replication of SARSCoV-2 in the throat, particularly during the first five days of symptoms. This was proven by the existence of viral RNA in the specimens. It was confirmed that viral mRNA is transcribed only in infected cells and is not packed into virions, thus suggesting the existence of lively infected cells in the specimens. ${ }^{41}$ Hence, if assuming that the throat functions as a major source of viral replication during the early stages of the viral course, proper oral hygiene measures in addition to usage of oral antiseptics could lower the viral replication affecting the viral load, thus not only reducing the transmission of the disease but also affecting its severity and seriousness. ${ }^{42,43}$

It was reported that the pathogenicity of SARS-CoV-2 relies on the capability of this virus to penetrate the cells, and there is logical proof that the transmembrane protein angiotensin-converting enzyme 2 (ACE2) is the main receptor for virus entry into the cell. ACE2 is expressed in the lungs, intestines, heart and kidneys, in addition to the oral mucosa and salivary gland. Thus, oral tissues could act as a reservoir for SARS-CoV-2, developing a high viral load in the oral cavity (saliva and crevicular fluid). ${ }^{44}$ This has been tackled in dental practices by antimicrobial povidone iodine pre-rinses that showed promising results in terms of viral killing. ${ }^{45}$ Moreover, Yoon et al. (2020) reported that chlorhexidine mouthwash was effective in reducing SARS-CoV-2 viral load in the saliva for a short-term period. ${ }^{46}$

In the current work, subgroup analysis of serious COVID-19 cases according to date of deterioration showed that $23.75 \%$ of patients deteriorated during the first week of illness. However, $76.25 \%$ revealed deterioration in the second week. In both subgroups, the number of patients with poor oral health status was significantly higher than those with good oral health status, which suggests that oral health may play a role in COVID-19 deterioration either due to viral infection or secondary bacterial infection. ${ }^{36}$

In the present study, CRP values were significantly higher in severe COVID-19 patients. This is in accordance with recent studies revealing that CRP levels may reflect the severity of COVID-19 disease. ${ }^{47,48} \mathrm{CRP}$ serves as an early marker of infection and inflammation. The normal concentration of CRP in the blood is less than $10 \mathrm{mg} / \mathrm{L}$; however, it rises rapidly and gives the highest peak in 48 hours from the disease onset. ${ }^{49}$ Although CRP is a nonspecific tool to differentiate between either viral and bacterial pneumonia or infectious and noninfectious conditions, several trials have shown a statistical significance between high CRP values and severity of the infection..$^{50,51,52}$ ${ }^{52}$ reover, CRP levels have been shown to hold prognostic importance during infections. ${ }^{51}$

In our present work, we investigated the correlation between oral health status and CRP values in the first week of COVID-19 diagnosis. We hypothesised that good oral hygiene could decrease viral load as well as secondary bacterial infections and hence decrease the severity of illness. The findings in our study supported this hypothesis, where significantly higher CRP values $(>18 \mathrm{mg} / \mathrm{L}$ ) were observed in participants with poor oral health $(\mathrm{p}<0.001)$. Moreover, CRP values significantly decreased in patients with good oral health $(\mathrm{p}<0.001)$. This is in agreement with several studies where high CRP values indicated more severe COVID-19 illness. ${ }^{47,48}$

Moreover, the recovery period was significantly delayed (six weeks) in those with poor oral health, while patients with good oral health had a faster recovery $(p<0.001)$, confirming the aforementioned hypothesis that oral health status could greatly impact the severity of the disease. However, some participants who had mild COVID-19 illness had a delayed recovery period, which can be explained from the literature by viral asthenia. ${ }^{53}$ In this oral health questionnaire, a cut-off value was estimated for the impact of oral health on COVID-19 severity, showing 71\% sensitivity (true positive cases) and $80 \%$ specificity (true negative cases). By this cutoff value, this detailed questionnaire evaluating oral health could be used on a larger scale for prediction of COVID-19 severity.

Finally, we can conclude that these types of questionnaires can introduce oral telemedicine, which could be applicable in future dental medicine since dental care is often invasive and requires aerosolisation, and dentists are always at higher risk of exposure to saliva. This is in line with several studies which recommend the reduction of face-to-face dental consultations to decrease the risk of infection and humanto-human transmission of diseases, especially SARS-COV-2. ${ }^{54,55}$

\section{Limitations}

The research team were unable to perform a dental examination due to quarantine restrictions and so a substitute oral health questionnaire was used; however, the authors acknowledge its limitations, in addition to confounding factors and limited study sample size. Moreover, COVID-19 severity is not only associated with respiratory disease and/or secondary infections, but can also be related to vascular injury or cytokine storm which were not included in the questions as they require a clinical examination. Furthermore, CRP is nonspecific and obtaining serial CRP levels during the full course of COVID-19 for each participant could not be achieved. However, some trials supported that the CRP value in the first week of illness could predict the disease prognosis. ${ }^{51,52}$ Despite these limitations, the findings of this study identify a future area for further research.

\section{Conclusions}

We concluded that oral health status could have a potential impact on the severity of COVID19 illness. Furthermore, poor oral health was correlated to increased values of CRP during the first week of illness, reflecting a serious condition of the disease. Moreover, a delayed recovery period was observed in patients with poor oral health. The cut-off value obtained from the oral health scoring used in this questionnaire could be used on a larger scale for the prediction of COVID-19 severity. In the future, this type of questionnaire could be used before the clinical examination to reduce the exposure time for dental practitioners. 
Conflict of interest

The authors declare that they have no conflict of interest. No financial aid or support was received.

\section{Acknowledgements}

The authors thank the study participants for their contribution. We express our appreciation and supportiveness to all frontline healthcare workers during the COVID-19 pandemic.

\section{Author contributions}

Amany Hany Mohamed Kamel: conceptualisation, data curation, formal analysis, funding acquisition, investigation, methodology, project administration, resources, software, supervision, validation, visualisation, roles/writing - original draft, writing - review and editing. Ahmed Basuoni: conceptualisation, data curation, formal analysis, funding acquisition, investigation, methodology, project administration, resources, software, supervision, validation, visualisation, roles/writing original draft, writing - review and editing. Zeinab A. Salem: data curation, investigation, project administration, software, supervision, validation, visualisation, writing - review and editing. Nermeen AbuBakr: conceptualisation, data curation, formal analysis, funding acquisition, investigation, methodology, project administration, resources, software, supervision, validation, visualisation, roles/ writing - original draft, writing - review and editing.

\section{References}

1. World Health Organisation. Novel coronavirus (2019-nCoV) situation report 22. 2020. Available online at https://apps. who.int/iris/handle/10665/330991 (accessed April 2020).

2. World Health Organisation. Coronavirus disease (COVID-19) pandemic. 2020. Available at https://www. who.int/emergencies/diseases/novel-coronavirus-2019 (accessed April 2020)

3. World Health Organisation. Coronavirus Disease (COVID-19) Dashboard. 2020. Available online at https://covid19.who.int/ (accessed January 2021).

4. Wang Y, Wang Y, Chen Y, Qin Q. Unique epidemiological and clinical features of the emerging 2019 novel coronavirus pneumonia (COVID-19) implicate special control measures. J Med Virol 2020; 92: 568-576.

5. Sinha P, Matthay M A, Calfee C S. Is a "Cytokine Storm" Relevant to COVID-19? JAMA Intern Med 2020; 180: 1152-1154.

6. Tay M, Poh C, Rénia L, MacAry P, Ng L. The trinity of COVID-19: immunity, inflammation and intervention. Nat Rev Immuno/ 2020; 20: 363-374.

7. Lia Q, Dinga $X$, Xiab G et al. Eosinopenia and elevated C-reactive protein facilitate triage of COVID-19 patients in fever clinic: A retrospective case-control study. EClinicalMedicine 2020; DOI: 10.1016/j. eclinm.2020.100375.

8. Huang C, Wang Y, Li X et al. Clinical features of patients infected with 2019 novel coronavirus in Wuhan, China. Lancet 2020; 395: 497-506.

9. Centers for Disease Control and Prevention. CDC updates, expands list of people at risk of severe COVID-19 illness. 2020. Available at https://www.cdc. gov/media/releases/2020/p0625-update-expandscovid-19.html (accessed January 2021)

10. Olsen I, Yamazaki K. Can oral bacteria affect the microbiome of the gut? J Oral Microbiol 2019; DOI: 10.1080/20002297.2019.1586422
11. Joshipura K, Ritchie C, Douglass C. Strength of Evidence Linking Oral Conditions and Systemic Disease. Compend Contin Educ Dent Suppl 2000; 30: 12-23; quiz 65.

12. Scannapieco F A. Role of Oral Bacteria in Respiratory Infection. J Periodontol 1999; 70: 793-802.

13. Imsand M, Janssens J P, Auckenthaler R, Mojon P, BudtzJørgensen E. Bronchopneumonia and oral health in hospitalised older patients. A pilot study. Gerodontology 2002; 19: 66-72.

14. Aas J A, Paster B J, Stokes L N, Olsen I, Dewhirst F E. Defining the normal bacterial flora of the oral cavity. J Clin Microbiol 2005; 43: 5721-5732.

15. Feng Y, Ling Y, Bai T et al. COVID-19 with different severities: a multi-centre study of clinical features. Am J Respir Crit Care Med 2020; 201: 1380-1388.

16. Fajnzylber J, Regan J, Coxen K et al. SARS-CoV-2 viral load is associated with increased disease severity and mortality. Nature Commun 2020; DOI: 10.1038/ s41467-020-19057-5.

17. Liu Y, Yan L, Wan L et al. Viral dynamics in mild and severe cases of COVID-19. Lancet Infect Dis 2020; 20: 656-657.

18. World Health Organisation. Egypt releases results of epidemiological study on oral health status. 2014. Available at http://www.emro.who.int/egy/egyptevents/results-of-epidemiological-study-on-oralhealth-status-released.html (accessed January 2021).

19. Levin L, Shpigel I, Peretz B. The use of a self-report questionnaire for dental health status assessment: a preliminary study. Br Dent J 2013; 214: E15.

20. Ruíz-López del Prado G, Blaya-Nováková V, SazParkinson Z et al. Design and validation of an oral health questionnaire for preoperative anaesthetic evaluation. Braz J Anesthesiol 2017; 67: 6-14.

21. Verity R, Okell L, Dorigatti I et al. Estimates of the severity of coronavirus disease 2019: a model-based analysis. Lancet Infect Dis 2020; 20: 669-677.

22. Wang D, Hu B, Hu C et al. Clinical Characteristics of 138 Hospitalized Patients With 2019 Novel CoronavirusInfected Pneumonia in Wuhan, China. JAMA 2020; 323 : 1061-1069.

23. World Health Organisation. Report of the WHO-China Joint Mission on Coronavirus Disease 2019 (COVID-19). 2020. Available at https://www.who.int/docs/defaultsource/coronaviruse/who-china-joint-mission-oncovid-19-final-report.pdf (accessed August 2020)

24. Worldometer. Coronavirus Symptoms (COVID-19). 2020. Available at https://www.worldometers.info/ coronavirus/coronavirus-symptoms/?fbclid=IWAR19 aftSD5oeads4bkyvmPfydVFhYjQLVUIAcgilCaJ7oFI4M (accessed April 2020).

25. Robert Koch Institut. Information and assistance for people with a higher risk of severe COVID-19 disease 2020. Available at https://www.rki.de/DE/Content/ InfAZ/N/Neuartiges_Coronavirus/Risikogruppen.htm (accessed June 2020).

26. NICE. COVID-19 rapid guideline: managing suspected or confirmed pneumonia in adults in the community Treatment and care planning. 2020. Available online at https://www.nice.org.uk/guidance/ng165/chapter/2Treatment-and-care-planning (accessed August 2020).

27. Greenhalgh T, Koh G, Car J. Covid-19: a remote assessment in primary care. BMJ 2020; DOI: 10.1136/ bmj.m1182.

28. The Centre for Evidence-Based Medicine. Are there any evidence-based ways of assessing dyspnoea (breathlessness) by telephone or video. 2020. Available at https://www.cebm.net/covid-19/are-there-anyevidence-based-ways-of-assessing-dyspnoeabreathlessness-by-telephone-or-video/ (accessed Apri 2020).

29. Chorin E, Padegimas A, Havakuk 0 et al. Assessment of Respiratory Distress by the Roth Score. Clin Cardiol 2016; 39: 636-639.

30. Loesche W J. Association of the oral flora with important medical diseases. Curr Opin Periodontol 1997; 4: 21-28.

31. Van Dyke TE, Dowell Jr V R, Offenbacher S, Snyder W, Hersh T. Potential role of microorganisms isolated from periodontal lesions in the pathogenesis of inflammatory bowel disease. Infect Immun 1986; 53: 671-677.

32. Page $R C$. The pathobiology of periodontal diseases may affect systemic diseases: inversion of a paradigm. Ann
Periodontol 1998; 3: 108-120

33. Genco R J. Periodontal disease and risk for myocardial infarction and cardiovascular disease Cardiovasc Rev Rep 1998; 19: 34-37.

34. Wang $S$, Lin D, Yang X et al. Prognostic value of C-reactive protein in patients with COVID-19. Infect Dis 2020: 9: 2445-2453.

35. Chakraborty S. Metagenome of SARS-Cov2 patients in Shenzhen with travel to Wuhan shows a wide range of species - Lautropia, Cutibacterium, Haemophilus being most abundant - and Campylobacter explaining diarrhea. 2020. Available online at https://www.researchgate.net/ publication/340152514_Metagenome_of_SARSCov2_patients_in_Shenzhen_with_travel_to_ Wuhan shows a wide range of species autropia_Cutibacterium_Haemophilus_being_most abundant_-_and_Campylobacter_explaining_diarrhea (accessed January 2021).

36. Khan A A, Khan Z. COVID-2019 associated overexpressed Prevotella proteins mediated hostpathogen interactions and their role in coronavirus outbreak. Bioinformatics 2020; 36: 4065-4069.

37. Cox M, Loman N, Bogaert D, O'Grady J. Co-infections: potentially lethal and unexplored in COVID-19. Lancet Microbe 2020; DOI: 10.1016/S2666-5247(20)30009-4.

38. Zhang $\mathrm{H}$, Zhang $\mathrm{Y}$, Wu J et al. Risks and features of secondary infections in severe and critical ill COVID-19 patients. Emerg Microbes Infect 2020; 9: 1958-1964.

39. Paju S, Scannapieco F. Oral biofilms, periodontitis, and pulmonary infections. Oral Dis 2007; 13: 508-512.

40. Yang Y, Li W, Wang Z M, Sun G Y, Zhou P, Han $X \mathrm{~L}$. Clinical significance of interleukin- 6 and-8 in patients with chronic periodontal disease and acute exacerbation of chronic obstructive pulmonary disease. Zhonghua Kou Qiang Yi Xue Za Zhi 2018; 53: 312-317.

41. Wölfel R, Corman V M, Guggemos W et al. Virological assessment of hospitalized patients with COVID-2019. Nature 2020; 581: 465-469

42. Pujadas E, Chaudhry F, McBride R et al. SARS-CoV-2 viral load predicts COVID-19 mortality. Lancet Respir Med 2020; DOI: 10.1016/S2213-2600(20)30354-4.

43. Meister T, Brüggemann Y, Todt D et al. Virucidal Efficacy of Different Oral Rinses Against Severe Acute Respiratory Syndrome Coronavirus 2. J Infect Dis 2020; 222: 1289-1292.

44. Xu H, Zhong L, Deng J et al. High expression of ACE2 receptor of 2019-nCoV on the epithelial cells of oral mucosa. Int J Oral Sci 2020; 12: 8.

45. Parhar H S, Tasche K, Brody R M et al. Topical preparations to reduce SARS-CoV-2 aerosolization in head and neck mucosal surgery. Head Neck 2020; 42 : 1268-1272.

46. Yoon J G, Yoon J Song J Y et al. Clinical Significance of High SARS-CoV-2 Viral Load in the Saliva. J Korean Med Sci 2020; DOI: 10.3346/jkms.2020.35.e195

47. Gao Y, Li T, Han M et al. Diagnostic utility of clinical laboratory data determinations for patients with the severe COVID-19. J Med Virol 2020; 92: 791-796.

48. Wang L. C-reactive protein levels in the early stage of COVID-19. Med Mal Infect 2020; 50: 332-334.

49. Meselson M. Droplets and Aerosols in the Transmission of SARS-CoV-2. N Engl J Med 2020; 382: 2063

50. Marnell L, Mold C, Du Clos T. C-reactive protein: Ligands, receptors and role in inflammation. Clin Immunol 2005: 117: 104-111.

51. Sahu B, Kampa R, Padhi A, Panda A. C-reactive protein A promising biomarker for poor prognosis in COVID-19 infection. Clin Chim Acta 2020; 509: 91-94.

52. Wang G, Wu C, Zhang Q et al. C-Reactive Protein Level May Predict the Risk of COVID-19 Aggravation. Open Forum Infect Dis 2020; DOI: 10.1093/ofid/ofaa153.

53. Jones J F, Miller B D. The Postviral Asthenia Syndrome. In Kurstak E, Lipowski Z J, Morozov P V (eds) Viruses, Immunity, and Mental Disorders. pp 441-451. Boston, MA: Springer, 1986

54. Meng L, Hua F, Bian Z. Coronavirus Disease 2019 (COVID-19): Emerging and Future Challenges for Denta and Oral Medicine. J Dent Res 2020; 99: 481-487.

55. da Costa C, Peralta F, Ferreira de Mello A. How Has Teledentistry Been Applied in Public Dental Health Services? An Integrative Review. Telemed J E Health 2020; 26: 945-954. 